\title{
COVARIANT INTEGRAL QUANTIZATIONS AND THEIR APPLICATIONS TO QUANTUM COSMOLOGY
}

\author{
JeAn PierRe GaZeAu
}

\author{
Astroparticle and Cosmology, Univ Paris Diderot, Sorbonne Paris Cité, Paris 75205, France \\ correspondence: gazeau@apc.univ-paris7.fr
}

\begin{abstract}
We present a general formalism for giving a measure space paired with a separable Hilbert space a quantum version based on a normalized positive operator-valued measure. The latter are built from families of density operators labeled by points of the measure space. We especially focus on group representation and probabilistic aspects of these constructions. Simple phase space examples illustrate the procedure: plane (Weyl-Heisenberg symmetry), half-plane (affine symmetry). Interesting applications to quantum cosmology ("smooth bouncing") for Friedmann-Robertson-Walker metric are presented and those for Bianchi I and IX models are mentioned.
\end{abstract}

KEYWORDS: integral quantization; covariance; POVM; affine group; Weyl-Heisenberg group; coherent states; FRW model; smooth bouncing.

\section{INTRODUCTION}

Group theory is one of the favorite domains of J. Patera and P. Winternitz. This contribution to the celebration of the Jiri and Pavel 80th birthday emphasizes the role of group representation theory in a certain type of quantization procedures. These methods [1 9 are named (covariant) integral quantizations and offer a large scope of possibilities for mapping a classical mathematical model of a physical system to a quantum model. They are based on normalized positive operator-valued measures (POVM) and present at least two attractive advantages:

(1.) By employing all resources of integration and distributions, they are most appropriate when we have to deal with some geometric singularities.

(2.) They afford a semi-classical phase space portrait of quantum states and quantum dynamics together with a probabilistic interpretation.

Moreover, their recent applications to quantum cosmology [10 15] has yielded interesting results as:

- consistent quantum dynamics of isotropic, anisotropic non-oscillatory and anisotropic models of Universe;

- singularity resolution;

- unitary dynamics without boundary conditions;

- consistent semi-classical description of involved quantum dynamics.

The aim of the present article is to give an overview of the methods, their main characteristics, and their promising applications to early cosmology. In Section 2 we give a rapid survey of canonical quantization with the problems raised by this procedure, and we define a few basic requirements that any quantization procedure should fullfill. In Section 3 we define POVM-based integral quantizations of functions defined on a measure space, their subsequent semi-classical counterparts (i.e., lower symbols) and discuss their possibilities when they are compared with other methods. Covariant integral quantizations are then considered when the measure space is homogeneous for some group, and we give the example of the half-plane viewed as the affine group of the real line. Section 4 is devoted to another example, the integral quantization of functions on the plane viewed as a coset of the Weyl-Heisenberg group. In Section 5 we illustrate the method with recent applications to quantum cosmology where the initial singularity is regularized by using the affine integral quantization. Finally we sketch in Section 6 a more philosophical point of view on the approaches described in this contribution.

A large part of the content has already been published, as exemplified by the above citations. On the other hand the original side of the paper lies in the synthetic presentation of the method together with some new ideas like the general (and promising) construction sketched in Section 3.2

\section{What IS QUANTIZATION?}

\subsection{Canonical QuAntization}

The basic procedure of quantization of a classical mechanical model is well known and is named "canonical" (Heisenberg, Born, Jordan, Dirac, Weyl, etc). Starting from a phase space or symplectic manifold, e.g. $\mathbb{R}^{2}$, e.g. the phase space for the motion of particle on the line, it consists in the maps

$$
\mathbb{R}^{2} \ni(q, p), \quad\{q, p\}=1 \mapsto \text { self-adjoint }(Q, P),
$$

with the canonical commutation rule (ccr) $[Q, P]=$ $i \hbar I$, and

$$
f(q, p) \mapsto f(Q, P) \mapsto(\operatorname{Sym} f)(Q, P) .
$$


Beyond the ordering problem [16 18] raised by the second step (symmetrisation), one should keep in mind that $[Q, P]=i \hbar I$ holds true with self-adjoint $Q, P$, only if both have continuous spectrum $(-\infty,+\infty)$, and there is uniqueness of the solution, up to unitary equivalence (von Neumann). But then what about singular $f$, e.g., the angle $\arctan (p / q)$ ? What about barriers or other impassable boundaries? The motion on a circle? In a bounded interval? On the half-line? Despite their elementary aspects, these examples leave open many questions both on mathematical and physical levels, irrespective of the manifold quantization procedures, like Path Integral Quantization (Feynman, thesis, 1942), Geometric Quantization with Weyl (1927), Groenewold (1946), Kirillov (1961), Souriau (1966), Kostant (1970), Deformation Quantization with Moyal (1947), Bayen, Flato, Fronsdal, Lichnerowicz, Sternheimer (1978), Fedosov (1985), Kontsevich (2003), Coherent state or anti-Wick or Toeplitz quantization with Klauder (1961), Berezin (1974).

Of course, the canonical procedure is universally accepted in view of its numerous experimental validations, one of the most famous and simplest one going back to the early period of Quantum Mechanics with the quantitative prediction of the isotopic effect in vibrational spectra of diatomic molecules [19]. These data validated the canonical quantization, contrary to the Bohr-Sommerfeld ansatz (which predicts no isotopic effect). Nevertheless this does not prove that another method of quantization fails to yield the same prediction [3]. Moreover, as already mentioned above, the canonical quantization is difficult, if not impossible, in many circumstances. As a matter of fact, the canonical or the Weyl-Wigner integral quantization maps $f(q)$ to $f(Q)$ (resp. $f(p)$ to $f(P)$ ), and so are unable to cure any kind of classical singularity. Clearly, quantization procedures may be intractable when different phase space geometries and/or topologies are considered. Self-adjointness of basic operators is not guaranteed anymore.

\subsection{QUANTIZATION: MINIMAL REQUIREMENTS}

In our viewpoint, any quantization of a set $X$ (e.g., a phase space or something else) and functions on it should meet four basic requirements:

(1.) Linearity - it is a linear map

$$
\mathfrak{Q}: \mathcal{C}(X) \mapsto \mathcal{A}(\mathcal{H}), \quad \mathfrak{Q}(f) \equiv A_{f},
$$

where:

- $\mathcal{C}(X)$ is a vector space of complex-valued functions $f(x)$ on set $X$, i.e., a "classical" mathematical model;

- $\mathcal{A}(\mathcal{H})$ is a vector space of linear operators (ignoring domain limitations) in some complex Hilbert space $\mathcal{H}$, i.e, a "quantum" mathematical model.

(2.) Unity - The map (3) is such that the function $f=1$ is mapped to the identity operator $I$ on $\mathcal{H}$.
(3.) Reality - A real $f$ is mapped to a self-adjoint operator $A_{f}$ in $\mathcal{H}$ or, at least, a symmetric operator,

(4.) Covariance - If $X$ is endowed with symmetry, the latter should be preserved in a "certain" sense.

Of course, further requirements are necessarily added, depending on the mathematical structures equipping $X$ and $\mathcal{C}(X)$ (e.g., measure, topology, manifold, closure under algebraic operations, time evolution or dynamics, etc). Moreover, a physical interpretation should be advanced about measurement of spectra of classical $f \in \mathcal{C}(X)$ or quantum $A_{f} \in \mathcal{A}(\mathcal{H})$ to which are given the status of observables. Finally, an unambiguous classical limit of the quantum physical quantities should exist, the limit operation being associated to a change of scale.

\section{Integral QuAntization(s)}

\subsection{INTEGRAL QUANTIZATION: GENERAL SETTING AND POVM}

Let $(X, \nu)$ be a measure space. Suppose that there exists an $X$-labeled family of bounded operators on a Hilbert space $\mathcal{H}$ resolving the identity $I$ :

$$
X \ni x \mapsto \mathrm{M}(x) \in \mathcal{L}(\mathcal{H}), \quad \int_{X} \mathrm{M}(x) \mathrm{d} \nu(x)=I,
$$

where the equality holds in a weak sense. The case we are specially interested in occurs when the $\mathrm{M}(x)$ 's are positive semi-definite and unit trace, i.e., when

$$
\mathrm{M}(x) \equiv \rho(x) \quad \text { (density operator). }
$$

Then, if $X$ is a space with suitable topology, the map

$$
\mathcal{B}(X) \ni \Delta \mapsto \int_{\Delta} \rho(x) \mathrm{d} \nu(x)
$$

may define a normalized positive operator-valued measure $(\mathrm{POVM})$ on the $\sigma$-algebra $\mathcal{B}(X)$ of Borel sets.

The quantization of complex-valued functions $f(x)$ on $X$ is the linear map:

$$
f \mapsto A_{f}=\int_{X} \mathrm{M}(x) f(x) \mathrm{d} \nu(x),
$$

understood as the sesquilinear form,

$$
B_{f}\left(\psi_{1}, \psi_{2}\right)=\int_{X}\left\langle\psi_{1}|\mathrm{M}(x)| \psi_{2}\right\rangle f(x) \mathrm{d} \nu(x),
$$

defined on a dense subspace of $\mathcal{H}$. If $f$ is real and at least semi-bounded, and if the $\mathrm{M}(x)$ 's are positive operators, then the Friedrich's extension of $B_{f}$ univocally defines a self-adjoint operator. If $f$ is not semi-bounded, there is no natural choice of a selfadjoint operator associated with $B_{f}$. We then need more information on $\mathcal{H}$ to solve this subtlety.

Covariance implies symmetry. Symmetry suggests a mathematical group $G$. A symmetry structure on $X$ presupposes that some group $G$ acts on $X$. Our hypothesis here is that $X$ is an homogeneous space for $G$, which means that $X \sim G / H$ for $H$ a subgroup of $G$. We now examine two possible cases. 


\subsection{1. $X=G$ : QUANTIZING THE GROUP}

Let $G$ be a Lie group with left invariant Haar measure $\mathrm{d} \mu(g)$, and let $g \mapsto U(g)$ be a unitary irreducible representation (UIR) of $G$ in a Hilbert space $\mathcal{H}$. Let $\mathrm{M}$ be a bounded operator on $\mathcal{H}$. Suppose that the operator

$$
\mathrm{R}:=\int_{G} \mathrm{M}(g) \mathrm{d} \mu(g), \quad \mathrm{M}(g):=U(g) \mathrm{M} U(g)^{\dagger},
$$

is defined in a weak sense. From the left invariance of $\mathrm{d} \mu(g)$ the operator $\mathrm{R}$ commutes with all operators $U(g), g \in G$, and so, from Schur's Lemma, $\mathrm{R}=c_{\mathrm{M}} I$ with

$$
c_{\mathrm{M}}=\int_{G} \operatorname{tr}\left(\rho_{0} \mathrm{M}(g)\right) \mathrm{d} \mu(g),
$$

where the unit trace positive operator $\rho_{0}$ is chosen in order to make the integral convergent. If $c_{\mathrm{M}}$ is finite and not zero, then we get the resolution of the identity:

$$
\int_{G} \mathrm{M}(g) \mathrm{d} \nu(g)=I, \quad \mathrm{~d} \nu(g):=\mathrm{d} \mu(g) / c_{\mathrm{M}} .
$$

For this reason, the operator $\mathrm{M}$ to be $U$ transported will be designated (admissible) fiducial operator. The method becomes more apparent when the representation $U$ is square-integrable in the following sense (for example, it is the case for the affine group of the real line, see below). Suppose that there exists a density operator $\rho$ which is admissible in the sense that

$$
c_{\rho}=\int_{G} \mathrm{~d} \mu(g) \operatorname{tr}(\rho \rho(g))<\infty,
$$

with $\rho(g)=U(g) \rho U^{\dagger}(g)$. Then the resolution of the identity (11) holds with $\mathrm{M}(g)$ and $c_{\mathrm{M}}=c_{\rho}$. This allows a covariant integral quantization of complexvalued functions on the group

$$
\begin{gathered}
f \mapsto A_{f}=\int_{G} \rho(g) f(g) \mathrm{d} \nu(g), \quad \mathrm{d} \nu(g):=\frac{\mathrm{d} \mu(g)}{c_{\rho}}, \\
U(g) A_{f} U^{\dagger}(g)=A_{\mathcal{U}(g) f} \quad \text { (covariance) }
\end{gathered}
$$

where

$$
(\mathcal{U}(g) f)\left(g^{\prime}\right):=f\left(g^{-1} g^{\prime}\right)
$$

is the regular representation if $f \in L^{2}(G, \mathrm{~d} \mu(g))$. This leads to a non-commutative version of the original manifold structure for $G$.

Example: affine CS integral quantization In this example, the group $G$ is the affine group of the real line and is also viewed as the phase space for the motion on the half-line, i.e., the half-plane $\{(q, p) \in$ $\left.\mathbb{R}_{+}^{*} \times \mathbb{R}\right\}$. The group law is defined as

$$
(q, p)\left(q_{0}, p_{0}\right)=\left(q q_{0}, \frac{p_{0}}{q}+p\right)
$$

and the left invariant measure is the symplectic $\mathrm{d} q \mathrm{~d} p$. This group has two UIR's which are both square integrable. We choose the following one.

$$
L^{2}\left(\mathbb{R}_{+}^{*}, \mathrm{~d} x\right) \ni \psi(x) \mapsto(U(q, p) \psi)(x)=\frac{e^{\mathrm{i} p x}}{\sqrt{q}} \psi\left(\frac{x}{q}\right)
$$

As a density operator we choose the rank one projector $\rho=|\psi\rangle\langle\psi|$ where the fiducial vector, i.e., the "wavelet" $\psi$ is in $L^{2}\left(\mathbb{R}_{+}, \mathrm{d} x\right) \cap L^{2}\left(\mathbb{R}_{+}, \mathrm{d} x / x\right)$ and its affine transport produces the overcomplete family of affine coherent states (ACS)

$$
|q, p\rangle=U(q, p)|\psi\rangle \text {. }
$$

The ACS integral quantization reads as

$$
f(q, p) \mapsto A_{f}=\int_{\mathbb{R}_{+} \times \mathbb{R}} \frac{\mathrm{d} q \mathrm{~d} p}{c_{\rho}} f(q, p)|q, p\rangle\langle q, p|
$$

with $c_{\rho}=\int_{0}^{\infty}|\psi(x)|^{2} \frac{\mathrm{d} x}{x}$. More details and new developments are given in the recent [20]. Applications of this method to the resolution of the singularity problem on a quantum level for a series of cosmological models (FRW, Bianchi I, Bianchi IX), for which the volume - expansion pair variables form the above half-plane, are given in the recent papers [10 15] and will be summarised in Section 5

\subsection{2. $X=G / H$ : QUANTIZING A NON-TRIVIAL GROUP COSET}

In the absence of square-integrability over $G$ (the trivial illustration is the Weyl-Heisenberg group which underlies in particular the canonical quantization, see Section (4), there exists a definition of square-integrable representations with respect to a left coset manifold $X=G / H$, with $H$ a closed subgroup of $G$ (it is the center in the Weyl-Heisenberg case), equipped with a quasi-invariant measure $\nu$ [2]. For a global Borel section $\sigma: X \rightarrow G$ of the group, let $\nu_{\sigma}$ be the unique quasi-invariant measure defined by

$$
\mathrm{d} \nu_{\sigma}(x)=\lambda(\sigma(x), x) \mathrm{d} \nu(x),
$$

where $\lambda(g, x) \mathrm{d} \nu(x)=\mathrm{d} \nu\left(g^{-1} x\right)$ for $g \in G$ with $\lambda(g, x)$ obeying the cocycle condition $\lambda\left(g_{1} g_{2}, x\right)=$ $\lambda\left(g_{1}, x\right) \lambda\left(g_{2}, g_{1}^{-1} x\right)$. Let $U$ be a UIR which is square integrable $\bmod (H)$ with an admissible $\rho$, i.e. $c_{\rho}:=\int_{X} \operatorname{tr}\left(\rho \rho_{\sigma}(x)\right) \mathrm{d} \nu_{\sigma}(x)<\infty$, with $\rho_{\sigma}(x)=$ $U(\sigma(x)) \rho U(\sigma(x))^{\dagger}$. Then we have the resolution of the identity and the resulting quantization

$$
f \mapsto A_{f}=\frac{1}{c_{\rho}} \int_{X} f(x) \rho_{\sigma}(x) \mathrm{d} \nu_{\sigma}(x)
$$

Covariance holds here too in a certain sense (see [2, Chapter 11]).

Besides the Weyl-Heisenberg group, another example concerns the motion on the circle for which $G$ is the group of Euclidean displacements in the plane, i.e., the semi-direct product $\mathbb{R}^{2} \rtimes \mathrm{SO}(2)$, and the subgroup $H$ is isomorphic to $\mathbb{R} 22$. Other examples involve the relativity groups, Galileo, Poincaré $[2,1+1$ Anti de Sitter (unit disk and SU(1,1)) [21], $1+1$ and $3+1$ de Sitter 23$]$. 


\subsection{AN EXAMPLE OF CONSTRUCTION OF ORIGINAL OPERATOR M OR $\rho$}

Let $U$ be a UIR of $G$ and $\varpi(x)$ be a function on the coset $X=G / H$. Suppose that it allows to define a bounded operator $\mathrm{M}_{\sigma}^{\varpi}$ on $\mathcal{H}$ through the operatorvalued integral

$$
\mathbf{M}_{\sigma}^{\varpi}=\int_{X} \varpi(x) U(\sigma(x)) \mathrm{d} \nu_{\sigma}(x) .
$$

Then, under appropriate conditions on the "weight" function $\varpi(\sigma(x))$ such that $U$ be a UIR which is square integrable $\bmod (H)$ and $\mathbf{M}_{\sigma}^{\varpi}$ is admissible in the above sense, the family of transported operators $\mathbf{M}_{\sigma}^{\varpi}(x):=U(\sigma(x)) \mathbf{M}_{\sigma}^{\varpi} U^{\dagger}(\sigma(x))$ resolves the identity.

\subsection{SEMI-CLASSICAL PORTRAITS}

Some quantization features, e.g., spectral properties of $A_{f}$, may be derived or at least well grasped from functional properties of the lower (Lieb) or covariant (Berezin) symbol (it generalizes Husimi function or Wigner function)

$$
A_{f} \mapsto \check{f}(x):=\operatorname{tr}\left(\mathrm{M}(x) A_{f}\right),
$$

When $\mathrm{M}=\rho$ (density operator) this new function is the local average of the original $f$ with respect to the probability distribution $\operatorname{tr}\left(\rho(x) \rho\left(x^{\prime}\right)\right)$

$$
f(x) \mapsto \check{f}(x)=\int_{X} f\left(x^{\prime}\right) \operatorname{tr}\left(\rho(x) \rho\left(x^{\prime}\right)\right) \mathrm{d} \nu\left(x^{\prime}\right) .
$$

The Bargmann-Segal-like map $f \mapsto \check{f}$ which, generalizes the Berezin or heat kernel transform when $X=G$ is a Lie group, is in general a regularization of the original, possibly extremely singular, $f$, and the classical limit itself means that, given one or more scale parameter $(\mathrm{s}) \epsilon_{(i)}$ and a distance $d(f, \check{f})$, we have

$$
d(f, \check{f}) \rightarrow 0 \quad \text { as } \quad \epsilon_{(i)} \rightarrow 0 .
$$

\subsection{Comments}

Beyond the freedom (think of analogy with Signal Analysis where different techniques are complementary) allowed by integral quantization, the advantages of the method with regard to other quantization procedures in use are of four types.

(i) The minimal amount of constraints imposed on the classical objects to be quantized.

(ii) Once a choice of (positive) operator-valued measure has been made, which must be consistent with experiment, there is no ambiguity in the issue, contrarily to other method(s) in use (think in particular of the ordering problem). To one classical model corresponds one and only one quantum model. Of course different choices of $(\mathrm{P}) \mathrm{OVM}$ are requested to be physically equivalent, e.g., leading to the same experimental predictions, if they are aimed to describe one specific system.
(iii)The method produces in essence a regularizing effect, at the exception of certain choices, like the Weyl-Wigner (i.e., canonical) integral quantization.

(iv)The method, through POVM choices, offers the possibility to keep a fully probabilistic content. As a matter of fact, the Weyl-Wigner integral quantization does not rest on a POVM.

\section{WEYL-HEISENBERG COVARIANT INTEGRAL QUANTIZATION $(\mathrm{S})$}

The Weyl-Heisenberg group is defined as $G_{\mathrm{WH}}=$ $\{(s, z) \mid s \in \mathbb{R}, z \in \mathbb{C}\}$ with multiplication law

$$
(s, z)\left(s^{\prime}, z^{\prime}\right)=\left(s+s^{\prime}+\operatorname{Im}\left(z \bar{z}^{\prime}\right), z+z^{\prime}\right)
$$

Let $\mathcal{H}$ be a separable (complex) Hilbert space with orthonormal basis $e_{0}, e_{1}, \ldots, e_{n} \equiv\left|e_{n}\right\rangle, \ldots$, and corresponding lowering and raising operators defined in the usual way

$$
\begin{aligned}
a\left|e_{n}\right\rangle & =\sqrt{n}\left|e_{n-1}\right\rangle, & a\left|e_{0}\right\rangle & =0, \\
a^{\dagger}\left|e_{n}\right\rangle & =\sqrt{n+1}\left|e_{n+1}\right\rangle, & {\left[a, a^{\dagger}\right] } & =I,
\end{aligned}
$$

It is well known that the Weyl-Heisenberg group has a unique non-trivial UIR $U$, up to unitary equivalence, each element in this equivalence class corresponding to a non-zero real number like the Planck constant. Consider the center $C=\{(s, 0) \mid s \in \mathbb{R}\}$ of $G_{\mathrm{WH}}$. Then, the set $X$ is the coset $X=G_{\mathrm{WH}} / C \sim \mathbb{C}$ with measure $\mathrm{d}^{2} z / \pi$. Choosing the trivial section $\mathbb{C} \ni z \mapsto$ $\sigma(z)=(0, z)$, to each $z$ corresponds the (unitary) displacement $(\sim$ Weyl $)$ operator

$$
\begin{gathered}
D(z):=U(\sigma(z))=e^{z a^{\dagger}-\bar{z} a}, \\
D(-z)=(D(z))^{-1}=D(z)^{\dagger} .
\end{gathered}
$$

The noncommutativity of quantum mechanics is encoded in the relation

$$
\begin{aligned}
D(z) D\left(z^{\prime}\right)=e^{\frac{1}{2}\left(z \overline{z^{\prime}}-\bar{z} z^{\prime}\right)} D\left(z+z^{\prime}\right) & \\
= & e^{\left(z \overline{z^{\prime}}-\bar{z} z^{\prime}\right)} D\left(z^{\prime}\right) D(z),
\end{aligned}
$$

i.e., $z \mapsto D(z)$ is a projective representation of the abelian group $\mathbb{C}$. The standard (i.e., SchrödingerKlauder-Glauber-Sudarshan) CS are defined as

$$
|z\rangle=D(z)\left|e_{0}\right\rangle \text {. }
$$

We now adopt the construction sketched in Section 3.2 . Let $\varpi(z)$ be a function on the complex plane obeying $\varpi(0)=1$. Suppose that it allows to define a fiducial operator $\mathrm{M}^{\varpi}$ on $\mathcal{H}$ through the operator-valued integral

$$
\mathrm{M}^{\varpi}=\int_{\mathbb{C}} \varpi(z) D(z) \frac{\mathrm{d}^{2} z}{\pi} .
$$

Then, the family of displaced $M^{\varpi}(z) \quad:=$ $D(z) \mathrm{M}^{\varpi} D(z)^{\dagger}$ under the unitary action $D(z)$ resolves the identity

$$
\int_{\mathbb{C}} \mathrm{M}^{\varpi}(z) \frac{\mathrm{d}^{2} z}{\pi}=I .
$$


It is a direct consequence of $D(z) D\left(z^{\prime}\right) D(z)^{\dagger}=$ $e^{z \bar{z}^{\prime}-\bar{z} z^{\prime}} D\left(z^{\prime}\right)$, of $\int_{\mathbb{C}} e^{z \bar{\xi}-\bar{z} \xi} \frac{\mathrm{d}^{2} \xi}{\pi}=\pi \delta^{2}(z)$, and of $\varpi(0)=1$ with $D(0)=I$. The resulting quantization map is given by

$$
\begin{aligned}
f \mapsto A_{f}^{\varpi}=\int_{\mathbb{C}} \mathrm{M}^{\varpi}(z) f(z) \frac{\mathrm{d}^{2} z}{\pi} \\
\quad=\int_{\mathbb{C}} \varpi(z) D(z) \overline{\mathfrak{f}_{\mathfrak{s}}}[f](z) \frac{\mathrm{d}^{2} z}{\pi},
\end{aligned}
$$

where are involved the symplectic Fourier transforms $\mathfrak{f}_{\mathfrak{s}}$ and its space reverse $\overline{\mathfrak{f}_{\mathfrak{s}}}$

$$
\mathfrak{f}_{\mathfrak{s}}[f](z)=\int_{\mathbb{C}} e^{z \bar{\xi}-\bar{z} \xi} f(\xi) \frac{\mathrm{d}^{2} \xi}{\pi}, \quad \overline{\mathfrak{f}_{\mathfrak{s}}}[f](z)=\mathfrak{f}_{\mathfrak{s}}[f](-z)
$$

Both are unipotent $\mathfrak{f}_{\mathfrak{s}}\left[\mathfrak{f}_{\mathfrak{s}}[f]\right]=f$ and $\overline{\mathfrak{f}_{\mathfrak{s}}}\left[\overline{\mathfrak{f}_{\mathfrak{s}}}[f]\right]=f$.

We have the following covariance properties

- Translation covariance:

$$
A_{f\left(z-z_{0}\right)}^{\varpi} D\left(z_{0}\right) A_{f(z)}^{\varpi} D\left(z_{0}\right)^{\dagger} .
$$

- Parity covariance

$$
A_{f(-z)}^{\varpi}=\mathrm{P} A_{f(z)}^{\varpi} \mathrm{P} \forall f \Longleftrightarrow \varpi(z)=\varpi(-z) \forall z,
$$

where $\mathrm{P}=\sum_{n=0}^{\infty}(-1)^{n}\left|e_{n}\right\rangle\left\langle e_{n}\right|$ is the parity operator.

- Complex conjugation covariance

$$
A_{f(z)}^{\varpi}=\left(A_{f(z)}^{\varpi}\right)^{\dagger} \forall f \Longleftrightarrow \overline{\varpi(-z)}=\varpi(z) \forall z,
$$

- For rotational covariance we define the unitary representation $\theta \mapsto U_{\mathbb{T}}(\theta)$ of $\mathbb{S}^{1}$ on the Hilbert space $\mathcal{H}$ as the diagonal operator

$$
U_{\mathbb{T}}(\theta)\left|e_{n}\right\rangle=e^{i(n+\nu) \theta}\left|e_{n}\right\rangle,
$$

where $\nu$ is arbitrary real. From the matrix elements of $D(z)$ one proves easily the rotational covariance property

$$
U_{\mathbb{T}}(\theta) D(z) U_{\mathbb{T}}(\theta)^{\dagger}=D\left(e^{i \theta} z\right)
$$

and its immediate consequence on the nature of $\mathrm{M}^{\varpi}$ and the covariance of $A_{f}^{\varpi}$ : the equality

$$
U_{\mathbb{T}}(\theta) A_{f}^{\varpi} U_{\mathbb{T}}(-\theta)=A_{T(\theta) f}^{\varpi},
$$

with $T(\theta) f(z):=f\left(e^{-i \theta} z\right)$, holds true if and only if

$$
\varpi\left(e^{i \theta} z\right)=\varpi(z) \forall z, \theta,
$$

i.e., if and only if $\mathrm{M}^{\varpi}$ is diagonal.

\section{Applications of ACS QUANTIZATION IN QUANTUM COSMOLOGY}

Let us consider the Friedmann-Robertson-Walker model filled with barotropic fluid with equation of state $\mathrm{p}=w \rho$. The line element is given by:

$$
d s^{2}=-N(t)^{2} d t^{2}+a(t)^{2} \delta_{i j} \omega^{i} \omega^{j},
$$

where $a(t)$ is the scale factor and the $\omega^{i}$ 's are rightinvariant dual vectors. The resolving of the Hamiltonian constraint leads to a model of singular universe analogous to a particle moving on the half-line $(0, \infty)$. Indeed, canonical coordinates $(q, p)$ can be introduced in terms of the volume $V \sim a^{3}$ and the expansion rate $\dot{V} / V \sim \dot{a} / a$ through the relations $V=q^{2 /(1-w)}$ and $\mathrm{K}=(3 / 8)(1-w) p q^{-(1+w) /(1-w)}$. Clearly, $q>0$ and $p \in \mathbb{R}$. Then the reduced Hamiltonian reads as

$$
\{q, p\}=1, \quad h(q, p)=\alpha(w) p^{2}+6 \tilde{k} q^{\beta(w)}, \quad q>0 .
$$

with $\tilde{k}=\left(\int \mathrm{d} \omega\right)^{2 / 3} k, \alpha(w)=3(1-w)^{2} / 32$ and $\beta(w)=$ $2(3 w+1) /(3(1-w)) . k=0,-1$ or 1 (in suitable unit of inverse area) depending on whether the universe is flat, open or closed. Assuming a closed universe with radiation content $w=1 / 3$ and $k=+1$, the classical isotropy singularity is cured on the quantum level by using affine CS quantization [10. Indeed the latter regularizes the kinetic term by adding a repulsive centrifugal potential which prevents the system from reaching the value $q=0$. Precisely, with the choice of a fiducial vector $\psi$,

$$
\begin{aligned}
\mathrm{h}=\frac{1}{24} p^{2}+6 q^{2} \mapsto A_{\mathrm{h}}=\frac{1}{24} P^{2} & +\frac{K(\psi)}{24} \frac{1}{Q^{2}} \\
& +6 M(\psi) Q^{2}, \\
P \equiv-\mathrm{i} \frac{d}{d x}, \quad Q \phi(x) \equiv & x \phi(x) .
\end{aligned}
$$

The constants $K(\psi)$ and $M(\psi)$ are $>0$ for all $\psi$ and their appearance is due to the affine CS quantization. Moreover, for a $\psi$ such that $K(\psi) \geq \frac{3}{4}$ the quantum Hamiltonian $A_{\mathrm{h}}$ is essentially self-adjoint, giving a unique unitary evolution: there is no need of boundary conditions.

The semi-classical dynamics is ruled by the ACS mean value of the quantum Hamiltonian :

$$
\begin{aligned}
& \left\langle q, p\left|A_{\mathrm{h}}\right| q, p\right\rangle \\
& =c(\psi) \int_{\mathbb{R}_{+} \times \mathbb{R}} \mathrm{d} q^{\prime} \mathrm{d} p^{\prime}\left|\left\langle q^{\prime}, p^{\prime} \mid q, p\right\rangle\right|^{2} \mathrm{~h}\left(q^{\prime}, p^{\prime}\right),
\end{aligned}
$$

with a displacement of the equilibrium point of the potential at $Q_{\mathrm{eq}}^{4}=\frac{1}{144} \frac{K}{M}$. The constant $c(\psi)$ is $>0$ for all $\psi$. In Figure 1 contour plots of phase space trajectories for classical Hamiltonian $h(q, p)$ (left) and semi-classical Hamiltonian (right) defined by 40 are compared.

As the result of affine quantization and restoring arbitrariness on $w$ and $k$, we obtain two corrections to the Friedmann equation which reads in its semiclassical version as

$$
\left(\frac{\dot{a}}{a}\right)^{2}+c^{2} a_{P}^{2}(1-w)^{2} A \frac{1}{V^{2}}+B \frac{k c^{2}}{a^{2}}=\frac{8 \pi G}{3 c^{2}} \rho,
$$

where $A$ and $B$ are positive factor dependent on $\psi$ and can be adjusted at will in consistence with (so far very hypothetical!) observations. The first correction is the repulsive potential, which depends on the volume, 

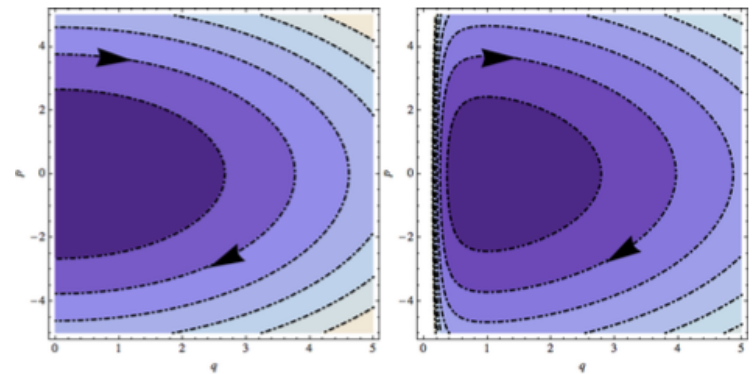

Figure 1. Contour plots of the classical and semiclassical Hamiltonians for the closed Friedmann universe. The singularity $q=0$ on the left is replaced with a bounce on the right.

and this excludes non-compact universes from quantum modelling. We notice that as the singularity is approached $a \rightarrow 0$, this potential grows faster $\left(\sim a^{-6}\right)$ than the density of fluid $\left(\sim a^{-3(1+w)}\right)$ and therefore at some point the contraction must come to a halt. Second, the curvature becomes dressed by the factor $B$. This effect could in principle be observed far away from the quantum phase. However, we do not observe the intrinsic curvature neither in the geometry nor in the dynamics of space. Nevertheless, for a convenient choice of $\psi$, this factor $\approx 1$

Also note that the form of the repulsive potential does not depend on the state of fluid filling the universe: the origin of singularity avoidance is quantum geometrical.

The applications of the same quantization methods to more involved cosmological models like Bianchi I and Bianchi IX are described in [11-15].

For other approaches to quantum cosmology based on affine symmetry see [24-26].

\section{Conclusion}

To conclude with an allusion to more "philosophical" perspectives, we think that integral quantization is just a part of a world of mathematical models for one phenomenon. Indeed, the physical laws are expressed in terms of combinations of mathematical symbols, and these combinations make sense for people who have learnt these elements of mathematical language. This language is in constant development, enhancement, since the set of phenomenons which are accessible to our understanding is constantly broadening. These combinations take place within a mathematical model. A model is usually scale dependent. It depends on a ratio of physical (i.e., measurable) quantities, like lengths, time(s), sizes, impulsions, actions, energies, etc. Changing scale for a model amounts to "quantize" or "de-quantize" toward (semi-)classicality. One changes perspective like one would change glasses to examine one's environment.

\section{ACKNOWLEDGEMENTS}

The author is indebted to Jiri Patera and Pavel Winternitz for inviting him at many occasions to share their enthusiasm in search for symmetry. He is also indebted to H. Bergeron, E. Czuchry, and P. Małkiewicz, for having developed with them a large part of the material exposed in the present contribution.

\section{REFERENCES}

[1] J.-P. Gazeau, Coherent States in Quantum Physics, Wiley-VCH, Berlin, 2009.

[2] S. T. Ali, J.-P Antoine, and J.-P. Gazeau, Coherent States, Wavelets and their Generalizations 2d edition, Theoretical and Mathematical Physics, Springer, New York (2013), specially Chapter 11.

[3] H. Bergeron, J.-P. Gazeau, and A. Youssef, Are the Weyl and coherent state descriptions physically equivalent? Phys. Lett. A $\mathbf{3 7 7}$ 598(2013); arXiv:1102.3556

[4] H Bergeron and J.-P Gazeau, Integral quantizations with two basic examples, Annals of Physics (NY) 344 43 (2014); arXiv: 1308.2348

[5] H. Bergeron, E. M. F. Curado, J.-P. Gazeau, and Ligia M. C. S. Rodrigues, Quantizations from (P)OVM's, J. Phys.: Conf. Ser. (2014); arXiv:1310.3304

[6] M. Baldiotti, R. Fresneda, and J.-P. Gazeau, Three examples of covariant integral quantization, Proceedings of Science (2014).

[7] J.-P. Gazeau and B. Heller, Positive-Operator Valued Measure (POVM) quantization, Axioms (Special issue on Quantum Statistical Inference) 41 (2015); http://www.mdpi.com/2075-1680/4/1/1

[8] M. Baldiotti, R. Fresneda, and J.-P. Gazeau, About Dirac\&Dirac constraint quantizations, Invited comment, Phys. Scr. 90074039 (2015).

[9] R. Fresneda and J.-P Gazeau, Integral quantizations with POVM and some applications, in Proceedings of the 30th International Colloquium on Group Theoretical Methods eds. J. Van der Jeugt et al, Journal of Physics: Conference Series 597 (2015) 012037.

[10] H. Bergeron, A. Dapor, J.-P. Gazeau, and P. Małkiewicz, Smooth big bounce from affine quantization, Phys. Rev. D 89083522 (2014); arXiv:1305.0653

[11] H Bergeron, A Dapor, J.-P. Gazeau, and P Małkiewicz, Smooth Bounce in Affine Quantization of Bianchi I, Phys. Rev D 91124002 (2015); arXiv:1501.07718

[12] H. Bergeron, E. Czuchry, J.-P. Gazeau, P. Małkiewicz, and W. Piechocki, Smooth quantum dynamics of the Mixmaster universe, Phys. Rev. D 92 061302(R) (2015); arXiv:1501.02174

[13] H. Bergeron, E. Czuchry, J.-P. Gazeau, P. Małkiewicz, and W. Piechocki, Singularity avoidance in quantum Mixmaster universe, Phys. Rev D 92124018 (2015); arXiv: 1501.07871

[14] H. Bergeron, E. Czuchry, J.-P. Gazeau, and P. Małkiewicz, Inflationary aspects of quantum Mixmaster universe, submitted; arXiv:1511.05790

[15] H. Bergeron, E. Czuchry, J.-P. Gazeau, and P. Małkiewicz, Vibronic framework for quantum Mixmaster universe, Phys. Rev D 93064080 (2016); arXiv:1512.00304 
[16] M. Born and P. Jordan, On quantum mechanics, Zs. f. Phys. 34858 (1925).

[17] B. S. Agarwal and E. Wolf, Calculus for Functions of Noncommuting Operators and General Phase-Space Methods in Quantum Mechanics, Phys. Rev. D 2 2161; 2187; 2206 (1970).

[18] F. A. Berezin and M. A. Shubin, The Schrödinger Equation, Kluwer Academic Publishers, Dordrecht, 1991.

[19] G. Herzberg, Molecular Spectra and Molecular Structure: Spectra of Diatomic Molecules, 2nd. ed., Krieger Pub., Malabar, FL, 1989.

[20] J.-P. Gazeau and R. Murenzi, Covariant Affine Integral Quantization(s), accepted to J. Math. Phys. (2016), arXiv:1512.08274

[21] J.-P. Gazeau and M. del Olmo, Unit disk \& SU(1,1) integral quantizations, in preparation.
[22] R. Fresneda, J.-P. Gazeau, and D. Noguera, Covariant integral quantization of the motion on the circle, in preparation.

[23] J.-P. Gazeau, A. Izadi, A. Rabei, and M. Takook, Covariant Integral Quantization of the motion on $1+1$ de Sitter space-time, in preparation.

[24] M. Fanuel and S. Zonetti, Affine Quantization and the Initial Cosmological Singularity, Eur. Phys. Lett. 101, 10001 (2013);

[25] J. R. Klauder, An Affinity for Affine Quantum Gravity, Proc. Steklov Institute of Mathematics 272, 169-176 (2011); gr-qc/1003.261

[26] J. R. Klauder, Enhanced Quantization, Particles, Fields \& Gravity, World Scientific, Singapore, 2015. 